\title{
CAMPI ELETTROMAGNETICI PURI A STRATI PARALLELI UNIFORMI,
}

di UMBERTO CISOTTI.

Sono puri, secondo il prof. Levi-Civita ${ }^{1}$ ), quei campi in cui il moto dell'elettricita avviene senza l'intervento di masse materiali e di legami cinematici fra le varie cariche.

In modo preciso, è quest'ultima la condizione che caratterizza i campi elettromagnetici puri, essendo ormai accettata in tutte le teorie degli elettroni l'ipotesi dell'assenza di materia ponderabite; soltanto in queste teorie le cariche non si considerano mai libere da vincoli cinematici ${ }^{2}$ ).

La considerazione dei campi elettromagnetici puri då origine ad un sistema differenziale che li caratterizza analiticamente.

$\dot{E}$ interessante la ricerca di soluzioni particolari di tale sistema, poichè dalla loro esistenza dipende la possibilità di movimenti spontanei delle cariche elettriche.

Il prof. Levi-Civita stesso ha messo in luce un primo esempio ${ }^{3}$ ), assegnando un tipo di soluzioni che corrispondono ad un trasporto per onde piane.

Lo stesso A. ha successiramente indicata una categoria di soluzioni che corrispondono a moti piani permanenti ${ }^{4}$ ).

In una recente Memoria la Sig." Gaffaratti ha studiato le soluzioni che competono a moti rigidi stazionari ${ }^{5}$ ).

1) Cfr. "Sur le mouvement de l'électricité sans liaisons ni fornes extérieures \$. (Compre: Rendus, 19 .1gosto 1907, pag. 417-4:0); e ancola dello stesso autore whi campi elettonmagnetiri puri domot, a rotı piani permanenti n. ( Itti del R. Ist. Vento di Sienze, Lettere ed Arti, 1908; Tonul LXVII, pag. 995-1010).

2) Vedi a tale proposito: Levi-livita. "Sull massa elettromagnetica .. (Nuovo Cimento, Ottribre 1907, p. 3-36; oppue Rivista di Scienza, 1907, Vol. II, N. IV, par. 387.412$)$.

3) Comptes Rexdus, loco citato.

4) Atti der R. Istituto Vereto, loco citato. 394).

5) "Sui campi elettromagnetici puri $\ldots$ (Nuovo Cimento, Maggio 1908, pag. 369- 
Nel presente lavoro tratto una categoria assai semplice di soluzioni aventi carattere stazionario.

Suppongo limitato da due piani paralleli l'ambiente $S$ in cui deve svolgersi il moto; e del fascio di piani da essi individuato, considero la porzione interna ad $\mathrm{S}$.

Mi propongo di cercare se sia possibile mantenere in $\mathrm{S}$ un campo elettromagnetico puro, libero da azioni esterne, mediante un movimento regolare di elettricita, in guisa che i parametri del campo, pur variando da piano a pianó, sieno costanti sopra un medesimo piano, in altre parole su ogni piano del fascio il campo sia uniforme.

E la risposta è affermativa.

Tutti i parametri del campo si possono far dipendere, in modo semplice, da due funzioni $H$ e $\lambda$, di una variabile indipendente. Esse devono soddisfare alle sole condizioni di essere finite e continue in un intervallo finito, e di più la prima di annullarsi negli estremi dell intervallo stesso.

In ogni piano del fascio si ha una indefinita traslazione uniforme, con velocita variabile, in grandezza e direzione, da piano a piano, ma mai inferiore, in valore assoluto, alla velocitá della luce.

Il campo non è generalmente trasversale rispetto alla direzione del moto delle cariche elettriche. Lo diviene nel caso particolare in cui tutte le cariche si muovano nella stessa direzione e con velocita eguale a quella della luce.

L'energia totale è (come nel caso tipico delle onde piane) metả di origine magnetica e metà di origine elettrica.

\section{1. - Richiamo delle equazioni caratteristiche di un campo elettromagnetico puro.}

Le equazioni caratteristiche di un campo elettromagnetico puro nel ruoto (o nei mezzi come l'aria) sono, con notazioni vettoriali manifeste,

le equazioni di Hertz:

$$
\mathrm{A} \frac{\partial \mathbf{E}}{\partial t}=-\operatorname{rot} \mathbf{H}-4 \pi \mathbf{A} \rho \mathbf{r}
$$




$$
\begin{aligned}
& \mathrm{A} \frac{\partial \mathbf{H}}{\partial t}=\operatorname{rot} \mathbf{E}, \\
& \operatorname{div} \mathbf{E}=4 \pi \rho, \\
& \operatorname{div} \mathbf{H}=0,
\end{aligned}
$$

che definiscono un campo elettromagnetico qualsiasi, e l'equazione di Lorentz ')

$$
\left.P[\mathbf{E}+\mathrm{A} \cdot \mathbf{H} \wedge \mathbf{v}]=0^{2}\right) .
$$

In queste

$$
\begin{aligned}
& \mathbf{E}=\text { forza elettrica, } \\
& \mathbf{H}=\text { forza magnetica, } \\
& \mathbf{v}=\text { velocità del flusso elettrico, }
\end{aligned}
$$

in un generico punto del campo che si considera; p rappresenta la densitá della distribuzione elettrica; infine $\mathrm{A}=\frac{1}{c}$, dove $c$ designa la velocità della luce nell' etere.

Le equazioni (I) - (V) valgono inquantochè si intenda di riferirsi ad un sistema sinistrorso, altrimenti bisognerebbe cambiare segno ai rot.

\section{2. - Dipendenza da una coordinata. - Flusso stazionario.}

- Comportamento qualitativo degli elementi caratteri. stici del campo sia entro che fuori della regione del moto.

Supponiamo, in particolare, che il moto dell'elettricita avvenga per piani paralleli in modo che i caratteri del campo sieno gli stessi per tutti i punti di uno stesso piano, pur variando da piano a piano.

Assunti i piani del fascio come piani $z=$ costante di un sistema di riferimento cartesiano, ortogonale, sinistrorso, la

1) Cfr. Levi-Civita, loc. cit.

2) II simbolo $\wedge$ desinna un prodntto vettoriale, conformemente alle propnste dei signori Marcolongo e Burali-Forti. (Cfr. Rend. del Circ. Mat. de Palermo, T. XXIV, 1907). 
ipotesi fatta viene analiticamente espressa dall' indipendenza di tutti i parametri del campo dalle coordinate $x$ e $y$.

Supporremo ulteriormente che il comportamento del fenomeno abbia carattere stazionario.

Ció implica che tutto sia indipendente da $t$.

Esplicitiamo le (I) - (V) in tali ipotesi. Poniamo breve. mente

$$
\mathbf{B}=\mathrm{A} \mathbf{v},
$$

con che B rappresenta la velocità del flusso rispetto a quella della luce.

Avremo

(I')

$$
\left\{\begin{array}{c}
\frac{d \mathrm{H}_{y}}{d z}=4 \pi \rho \beta_{x}, \\
\frac{d \mathrm{H}_{x}}{d z}=-4 \pi \rho \beta_{y}, \\
\beta_{z}=0 ;
\end{array}\right.
$$

$$
\begin{aligned}
& \frac{d \mathrm{E} x}{d z}=\frac{d \mathrm{E}_{y}}{d z}=0 ; \\
& \frac{d \mathrm{E}_{z}}{d z}=4 \pi \rho \\
& \frac{d \mathrm{H}_{z}}{d z}=0 ;
\end{aligned}
$$

$\left(V^{\prime}\right)$

$$
\begin{aligned}
& \rho\left[\mathrm{E} x-\mathrm{H}_{z} \beta_{y}\right]=0, \\
& \rho\left[\mathrm{E}_{y}+\mathrm{H}_{z} \beta_{x}\right]=0, \\
& \rho\left[\mathrm{F}_{z}+\left(\mathrm{H}_{x} \beta_{y}-\mathrm{H}_{y} \beta_{x}\right)\right]=0 .
\end{aligned}
$$

Sia $S$ la regione dello spazio in cui vì è effettivamente dell'elettricità, in cui ciò̀ $\rho$ è generalmente ') diver'so da zero.

Sia S limitato dai due piani

$$
\approx=l_{1} \text { e } \quad z=l_{2} \text {. }
$$

1) Colla parola generalmente si intende che $\rho$ possa annullarsi al più in punti, linee o superficie isolate. 
Ammetteremo che $\rho$ sia finita e continua entro $\mathbf{S}$, senza escludere che passi bruscamente a zero attraversando i piani limiti verso l'esterno.

Fuori di $S$ è naturalmente $\rho=0$.

Ammetteremo ancora che la forza elettrica $\mathbf{E}$ e la forza magnetica $\mathbf{H}$ sieno finite e continue tanto in $\mathbf{S}$ che fuori $\mathrm{e}$ attraverso i piani limiti.

3. - Integrazione del sistema (I') - (V').

Dalle (II') e (IV') scende che $\mathbf{E}_{x}, \mathbf{E}_{y}, \mathrm{H}_{z}$, hanno valore costante in tutto lo spazio.

Se si nota che per $z= \pm \infty$ il campo è a ritenersi nullo, si deve dire che

$$
\mathrm{E}_{x}=\mathrm{E}_{y}=\mathrm{H}_{z}=0 \quad \text { in tutto lo spazio. }
$$

Con ciò riescono identicamente soddisfatte le prime due delle $\left(V^{\prime}\right)$.

Nei punti esterni ad $S$ l'ultima delle ( $V^{\prime}$ ) è identicamente soddisfatta; nei punti internj, essendo, $\rho \neq 0$, dà la relazione

$$
\mathrm{E}_{z}=\mathrm{H}_{y} \beta_{x}-\mathrm{H}_{x} \beta_{y},
$$

ed eliminando da questa e dalle (I') e (III') le incognite $\mathbf{P} x$, $\boldsymbol{\beta}_{y}, \rho$ e ponendo brevemente $\mathrm{E}$ al posto di $\mathrm{E}_{z}$ si ottiene

$$
\frac{d \mathrm{~F}^{\mathrm{a}}}{d z}=\frac{\left.d\left(\mathrm{H}_{x^{3}}+\mathrm{H}_{y}\right)^{3}\right)}{d z}
$$

dalla quale integrando e notando che

$$
\mathrm{H}_{x^{2}}+\mathrm{H}_{y}{ }^{2}=\mathrm{H}^{2},
$$

si ricava la relazione

$$
\mathrm{E}^{2}-\mathrm{H}^{2}=\text { costante entro } \mathrm{S} \text {. }
$$

Dalle due prime delle (I') e dalla (III') scende che fuori di $\mathrm{S}$ (essendo $\mathrm{p}=0$ ), $\mathrm{H} x, \mathrm{H}_{y}, \mathrm{E}$ hanno valore costante; ma poichè, come abbiamo già notato, per' $^{\prime} z= \pm \infty$ il campo è a ritenersi nullo, sarà

$$
\mathrm{H}_{x}=\mathrm{H}_{y}=\mathrm{E}=0 \quad \text { fuori di } \mathrm{S} .
$$


Per la continuitá delle forze elettromagnetiche attraverso ai piani limiti di $\mathrm{S}$ saranno $\mathrm{E}$ ed $\mathrm{H}$ nulli sugli stessi. D'altra parte, per la (1), $\mathbf{E}^{2}$ e $\mathrm{H}^{2}$ differiscono nei punti interni per una quantitá costante che, essendo nulla sul contorno, sará nulla pure in tutti i punti interni; potremo scrivere pertanto $\mathbf{E}= \pm \mathbf{H}$ ovvero, potendosi sempre assumere come direzione positiva dell'asse $z$ quella della forza elettrica,

$$
\mathrm{E}=\mathrm{H} \text { entro } \mathrm{S} \text {. }
$$

Possiamo concludere che l' energia totale è metà di origine elettrica e metà di origine magnetica.

Dilla terza delle ( $\left.I^{\prime}\right)$ scende che le cariche elettriche non escono dal piano su cui giacciono inizialmente.

Inoltre, per le ipotesi ammesse, in ogni piano $z=$ costante, è costante la velocità in grandezza e direzione, sha cioè una indefinita traslazione uniforme.

La velocità varia, come ora vedremo, da piano a piano, in modo regolare, non scendendo mai, in valore assoluto, al di sotto della velocità della luce.

Infatti, moltiplicando ambo i membri della prima delle ( $\left.I^{\prime}\right)$ per $i=\boldsymbol{V}=\mathbf{I}$, e sommando l' equazione risultante colla seconda, avremo

$$
\frac{d}{d z}\left(\mathrm{H}_{x}+i \mathrm{H}_{y}\right)=4 \pi \rho i\left(\beta_{x}+i \beta_{y}\right) .
$$

Supponiamo che $H$ non si annulli mai nell' intervallo $\left(l_{1}, l_{2}\right)$, esclusi beninteso gli estremi; possiamo allora porre ${ }^{1}$ )

$$
\left\{\begin{array}{c}
\mathrm{H}=e^{h}, \\
\mathrm{H} x+i \mathrm{H}_{y}=\mathrm{H} e^{i \lambda}=e^{h+i \lambda}, \\
\beta_{x}+i \beta_{y}=\boldsymbol{\beta} e^{i \mu}
\end{array}\right.
$$

e per l'ipotesi fatta $h$ si mantiene finita nei punti interni del citato intervallo.

1) Se $\mathrm{H}$ si annullasse anche in un numero discreto di punti dell' intervallo $\left(l_{1}, l_{2}\right)$, basterebbe isolare tali punti, mediante degli intorn. In ciascuno dei rimanenti tıatti, mantecendosi $\mathrm{H}$ diverso da zelo, sarebbero lecite le posizioni ( 3$)$ e le conseguenti deduzioni. 
Dalle precedenti posizioni risulta che $\lambda$ e $\mu$ rappresentano rispettivamente le inclinazioni delle linee di forza magnetica e delle linee di flusso sull asse delle ascisse.

Per le (3) e la (III'), la (2) diviene, indicando con un apice la derivazione rispetto a $z$,

$$
\left(h^{\prime}+i \lambda^{\prime}\right) e^{h+i \lambda}=i h^{\prime} \beta e^{h+i \mu} .
$$

Non può essere $h^{\prime} . e^{h}$ identicamente nulla, poichè, per la prima delle (3), ció implicherebbe $\mathrm{H}=$ costante in tutto lo intervallo e quindi $\mathrm{H}=0$ (duvendo $\mathrm{H}$ annullarsi agli estremi) il chè tobbiamo escludere poichè, le (III') e (I'), darebbero $p=0$ entro $S$, contro l'ipotesi fondamentale; perciò sarà lecito dividere ambo i membri della precedente per $h^{\prime} e^{h+i \lambda}$ otterremo

$$
1+i \frac{\lambda^{\prime}}{h^{\prime}}=i B e^{i(\mu-\lambda)}
$$

Eguagliando le parti reali ed i coefficienti dell' immaginario, si hanno dalla precedente le due relazioni seguenti

$$
\left\{\begin{array}{l}
\beta \operatorname{sen}(\mu-\lambda)=-1, \\
\beta \cos (\mu-\lambda)=\frac{\lambda^{\prime}}{h^{\prime}} .
\end{array}\right.
$$

Da queste, quadrando e sommando, si ha

$$
\beta^{2}=1+\left(\frac{\lambda^{\prime}}{h^{\prime}}\right)^{2}
$$

e dividendo membro a membro

$$
\operatorname{tg}(\mu-\lambda)=-\frac{h^{\prime}}{\lambda^{\prime}}
$$

La (4) ci dice appunto che la velocità del fusso non è mai inferiore, in valore assoluto, a quella della luce.

Ia (5), che per la (4), può anche scriversi

$$
\operatorname{tg}(\mu-\lambda)=-\frac{1}{\sqrt{\beta^{2}-1}},
$$

ci dà l'inclinazione $\mu-\lambda$ delle linee di flusso sulle linee di forza magnetica. 
Da essa risulta che, in generale, il campo non è trasversale rispetto alla direzione delle cariche. Infatti l'jpotesi di un campo trasversale richiederebbe che fosse dappertutto $\beta^{2}=1$; nel qual caso, dalla (4), si deduce $\lambda=$ costante e quindi $\mu=$ costantè; dunque il campo è trasversale allora e solo allora che tutte le cariche si muovono nella stessa direzione, colla velocità della luce.

Riportando nella (4) al posto di $h$ la sua espressione $\log \mathrm{H}$, desunta dalla prima delle (3), avremo

$$
\boldsymbol{\beta}=\sqrt{1+\left(\frac{\lambda^{\prime}}{\left.\frac{d \log \mathrm{H}}{a}\right)^{2}}\right.}
$$

formola che ci dà il valore assoluto della velocità in funzione del valore assoluto e dell'inclinazione della forza magnetica.

Riassumendo

Sieno $\mathrm{H} e \mathrm{\lambda}$ due funzioni di $z$, finite $e$ continue nello intervallo $\left(l_{1}, l_{2}\right)$ di cui la prima si annulli agli estremi.

Se si prende a considerare il campo $\mathrm{S}$ limitato dai piani $z=l_{1}, z=l_{2}$, si possono esprimere, per mezzo di $\mathrm{H}$ e di $\lambda$, tutti $i$ parametri del campo nel modo seguente:

(6)

$$
\begin{aligned}
& \mathrm{H}_{x}=\mathrm{H} \cos \lambda, \mathrm{H}_{y}=\mathrm{H} \operatorname{sen} \lambda, \mathrm{H}_{z}=0 ; \\
& \mathrm{E}_{x}=\mathrm{E}_{y}=0, \mathrm{E}_{z}=\mathrm{E}=\mathrm{H}: \\
& \rho=\frac{1}{4 \pi} d \frac{\mathrm{H}}{d z} \\
& \beta=\sqrt{1+\left(\frac{\lambda^{\prime}}{d \log \mathrm{H}}\right)^{2}} ; \\
& \beta_{x}=\beta \cos \mu, \beta_{y}=\beta \operatorname{sen} \mu, \beta_{z}=0 \\
& \text { essendo } \mu \text { definita dalla } \\
& \operatorname{tg}(\mu-\lambda)=\overline{-1} \overline{\beta^{2}-1} .
\end{aligned}
$$

Queste formole valgono nei punti interni ad $\mathrm{s}$, incluso il contorno. 
All' esterno dis, dove non $c^{\prime} \dot{e}$ elettricit $\dot{a}, \dot{e}$

$$
\mathbf{E}=\mathbf{H}=0 \text {. }
$$

Esempio. - Sieno

$$
z=+l \text { e } z=-l
$$

i due piani che limitano $\mathrm{S}$, e poniamo, ad es.

$$
\mathrm{H}=l^{2}-z^{2}, \lambda=z^{2} \text {. }
$$

Come si vede, per $z= \pm l$, H si annulla.

Portando nelle (6) le precedenti espressioni, abbiamo

$$
\begin{aligned}
& \mathrm{H} x=\left(l^{2}-z\right)^{2} \cos z^{2}, \mathrm{H} y=\left(l^{2}-z^{2}\right) \operatorname{sen} z^{2} ; \\
& \mathrm{E}=l^{2}-z^{2} ; \\
& \rho=\frac{-z}{2 \pi} ; \\
& \beta=V \overline{1+\left(l^{2}-z^{2}\right)^{2}}, \\
& \mu=z^{2}-\operatorname{arctg} \frac{1}{\sqrt{\beta^{2}-1}}=z^{2}-\operatorname{arctg} \frac{1}{l^{2}-z^{2}} .
\end{aligned}
$$

Dalla precedente espressione di $\beta$, se si tiene presente che entro $\mathrm{S}$ è

$$
|z| \leq l
$$

si deduce che il massimo valore della velocità è $\boldsymbol{\beta}=\boldsymbol{V} \overline{1+l^{4}}$, ed è raggiunto dalle cariche che si muovono nel piano $(z=0)$, equidistante da quelli che limitano lo spazio S. Su questi piani limiti le cariche si muovono colla velocita della luce.

Come si vede tutti gli elementi che cal'atterizzano il campo $\mathrm{S}$ si mantengono regolari in $\mathrm{S}$ e soddisfanno al contorno e fuori di $S$ alle condizioni volute. 